The ASTROPHYSICAL JOURNAL SUPPLEMENT SERIES, 90:981-983, 1994 February

(C) 1994. The American Astronomical Society. All rights reserved. Printed in U.S.A.

\title{
BOHM DIFFUSION AND COSMIC-RAY-MODIFIED SHOCKS
}

\author{
PETER DUFFY \\ Max-Planck-Institut für Kernphysik, Heidelberg 103980 , Germany \\ Received 1993 March 3; accepted 1993 June 9
}

\begin{abstract}
A numerical solution to the problem of self-consistent diffusive shock acceleration is presented. The cosmic rays are scattered, accelerated and exert a back-reaction on the gas through their interaction with turbulence frozen into the local fluid frame. Using a grid with a hierarchical spacetime structure the physically interesting limit of Bohm diffusion ( $\kappa \propto p v$ ), which introduces a wide range of diffusion lengthscales and acceleration timescales, can be studied. Some implications for modified shocks and particle acceleration are presented.
\end{abstract}

Subject headings: acceleration of particles — cosmic rays - diffusion — shock waves

\section{INTRODUCTION}

The problem of the self-consistent evolution of astrophysical shock waves and cosmic rays (CRs) accelerated by the firstorder Fermi mechanism is one that was first stated by Axford, Blandford, \& Leer (1977). In that paper it was proposed that once such an acceleration mechanism becomes efficient the pressure of the CR themselves must influence the hydrodynamics. It was not until Drury \& Völk ( 1981 ) and Axford, Leer, \& McKenzie (1982) that this problem was addressed quantitatively in the steady state limit. By taking an appropriate moment of the CR transport equation these authors obtain an equation for the $\mathrm{CR}$ energy density, $E_{C}$. On this level the energetic particles are modeled as a nonviscous fluid with an extra diffusion term describing the scattering off magnetic turbulence that is frozen into the background flow. The gas dynamics is modified to include the pressure gradient of the CRs. While this approach may contain the important conceptual features of the interaction, it is open to the criticism that it ignores some important kinetic effects (Achterberg, Blandford, \& Periwal 1984 and Heavens 1984), since it leaves the energetic particle spectrum undetermined. This also applies to the numerical work on time-dependent two-fluid models (Dorfi 1990 and Jones \& Kang 1990).

The obvious way around this difficulty is to solve the full CR transport equation for the phase space density $f(x, p, t)$ from which $P_{C}$ and $E_{C}$ can be calculated. Falle \& Giddings (1987) and Bell (1987) first presented numerical solutions to self-consistent shock acceleration with a kinetic description for the CRs. These papers, and all subsequent work on this method, ignore self-consistent wave generation by the energetic particles and assume that there is sufficient turbulence present to cause diffusion. If, as is usual, the diffusion coefficient is taken to be proportional to some power of momentum, $\kappa \propto p^{\alpha}$, then $\alpha$ is an additional free parameter in the theory. Each of the two original numerical papers consider this exponent to be quite small, in the range of one-fourth to one-third. This proves to be necessary since a stronger dependence of $k$ on $p$ would introduce a wide range of diffusion lengthscales $(L(p) \sim \kappa(p) / U$ with $U$ the flow speed) and acceleration timescales $(\tau(p) \sim$ $\kappa(p) / U^{2}$ ) that are not easily accommodated in a finite difference method. However, in the presence of strong wave excitation we would be more justified in setting the mean free path of an energetic particle equal to its gyroradius in the background magnetic field which leads to $\kappa_{\mathrm{B}} \propto p v$, the Bohm limit. Until recently such a strongly energy-dependent $\kappa$ had only been used for Monte Carlo calculations as in Ellison, Möbius, \& Paschmann (1990) and Jones \& Ellison (1991) for a review. In this work a maximum energy is introduced beyond which energetic particles either escape from the system or cannot be scattered sufficiently to take part in the acceleration process. With both the thermal gas and the CRs assumed to undergo Bohm type diffusion the resulting self-consistent steady state solutions are calculated.

There are, however, some systems where the upper cutoff is determined more by the time available to accelerate particles than by geometrical factors. This seems to be the case in CRs produced by supernova remnants, Drury, Markiewicz, \& Völk (1989). In this paper a numerical method (Duffy 1992) is briefly described where such time-dependent systems can be solved with $\kappa_{\mathrm{B}}$.

\section{PHYSICAL MODEL}

In what follows it is assumed that the total particle distribution is divided into two distinct populations. The first is the thermal component which obeys the normal mass, momentum, and energy conservation laws for an inviscid fluid. A mean magnetic field is imbedded in this fluid along with MHD turbulence. The second, nonthermal, component has a much higher characteristic energy than an average thermal particle and is described on a kinetic level through its phase space distribution function (see Berezhko \& Krymsky 1988 for a review of shock acceleration). The process by which thermal particles are injected into the higher energy population is not yet understood. In the presence of a shock wave it is therefore described in the following simple manner. A fraction, $\eta$, of thermal particles incident on the shock front from upstream are injected as the lowest energy CR with a momentum of $p_{i}=\lambda m c$ where $m$ is the particle's mass. This corresponds to an injected energy flux of $I=0.5 \eta \lambda^{2} \rho_{1} U_{1} c^{2}$ with $\rho_{1} U_{1}$ the mass flux incident on the shock in the shock rest frame. In one dimension this coupled gas and CR system is described by the four equations

$$
\frac{D \rho}{D t}=-\rho \frac{\partial U}{\partial x}
$$




$$
\begin{aligned}
\frac{D U}{D t} & =-\frac{1}{\rho} \frac{\partial}{\partial x}\left(P_{G}+P_{C}\right) \\
\frac{D E_{G}}{D t} & =-\gamma_{G} E_{G} \frac{\partial U}{\partial x}-I \delta\left[x-x_{s}(t)\right], \\
\frac{D f}{D t}=\frac{\partial}{\partial x}\left(\kappa_{\mathrm{B}} \frac{\partial f}{\partial x}\right) & +\frac{1}{3} \frac{\partial U}{\partial x} p \frac{\partial f}{\partial p} \\
& +\frac{I}{4 \pi p_{i}^{2} T\left(p_{i}\right)} \delta\left(p-p_{i}\right) \delta\left[x-x_{s}(t)\right]
\end{aligned}
$$

with a nonrelativistic equation of state for the gas $P_{G}=\left(\gamma_{G}-\right.$ 1) $E_{G}, \gamma_{G}=\frac{5}{3}$. The CR transport equation (4) describes the adiabatic compression in a nonuniform flow and scattering off MHD waves. The CR "adiabatic exponent" $\gamma_{C}=1+P_{C} / E_{C}$ is calculated from the two integral moments

$$
\begin{aligned}
& P_{C}=\frac{4 \pi}{3} \int p^{3} v f(p) d p \text { and } \\
& E_{C}=4 \pi \int p^{2} T(p) f(p) d p
\end{aligned}
$$

A shock is produced by a piston driving into a gas that is initially uniform and at rest. The gas pressure and density is normalized by setting the far-upstream values to unity, while the ambient CR energy density is taken to be zero so that all of the energetic particles originate from the injection process.

The details of the numerical method are contained in Duffy (1992), and only the briefest of summaries is given here. Equations (1)-(3) are differenced explicitly, while a semi-implicit Crank Nicholson scheme is used for the CR transport equation. The important difference between this and previous work is that the spatial and temporal step sizes are doubled in successively coarser regions away from the shock upstream. The motivation behind such a hierarchical grid is that with $\kappa$ increasing substantially from $p_{i}$ up to the highest energy particles the CR distribution is increasingly dominated by high-energy particles upstream. Therefore the acceleration timescales and diffusion lengthscales are both larger for a typical particle farther upstream than for one near the shock. Thus the resolution, and hence the computational expense, may be decreased away from the discontinuity. This requires interior boundary conditions to ensure number conservation, etc., between adjoining regions.

\section{RESULTS}

As test case of a fast shock that becomes strongly modified by energetic particles the initial shock Mach number is set equal to 41 , while the level of injection is put at $\eta=0.002$. The injection momentum is given by $\lambda=0.1$. With the above initial and boundary conditions Figure 1 shows the evolution and time asymptotic quasi steady state of the coupled system with these parameter values.

The first panel shows the evolution of the CR adiabatic index at the shock with the unit of time taken to be $t_{0}$, the test particle acceleration timescale at $p_{i}$. It is evident from the level-

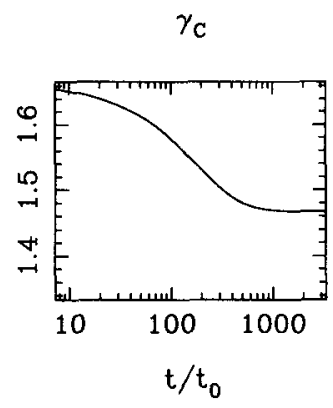

$P_{G}$ (solid) and $P_{C}$

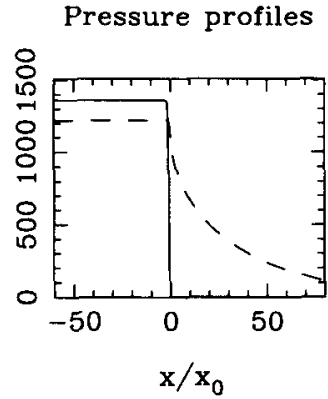

Gas velocity
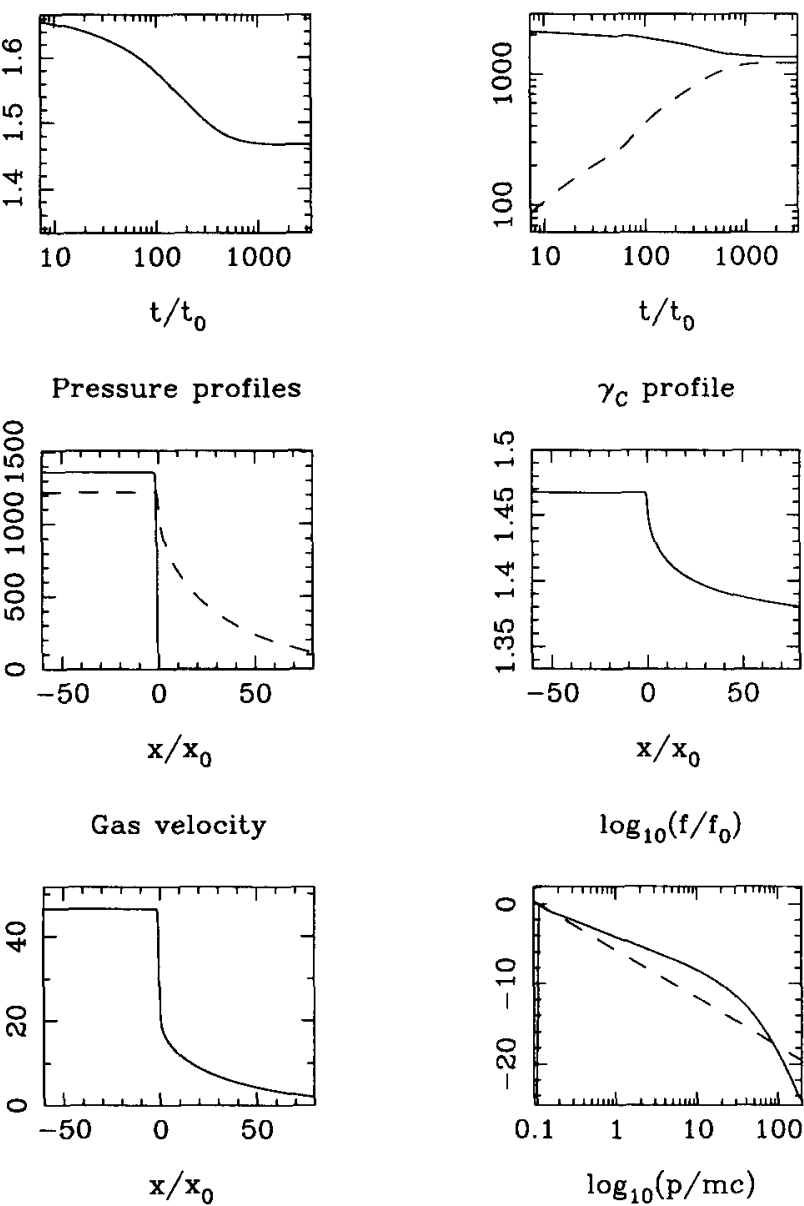

$\gamma_{C}$ profile
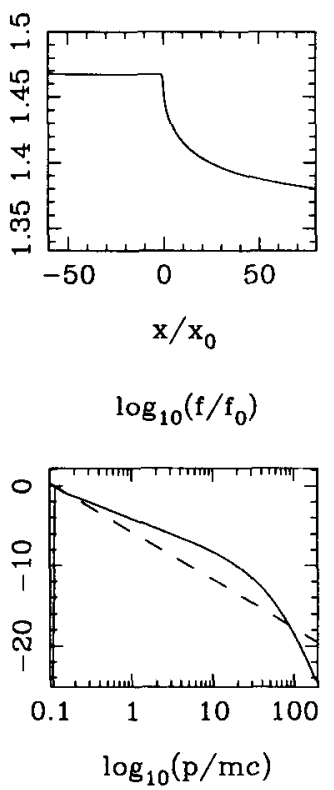

FIG. 1.-Evolution and final quasi steady states for a cosmic-raymodified shock and cosmic-ray spectrum with $M_{s}=41$ and $\eta=0.002$.

ing off of $\gamma_{C}$ at late times that although the spectrum may be still evolving it does so in such a manner that the CR energy density remains constant, a feature also discussed in Falle \& Giddings ( 1987) for $\kappa \propto p^{0.25}$. The second plot shows the decline and rise of the gas and CR pressures, respectively. The remaining panels display various profiles once this quasi steady state is reached with $x_{0}$ the test particle diffusion length scale at $p_{i}$. The broad diffusive tail of the upstream energetic particles in the third plot becomes dominated by the highest energy CRs. This is apparent from the profile of the local adiabatic index which falls off away from the shock toward $\frac{4}{3}$. The initial shock profile has now been modified to give a weaker subshock followed by a precursor region produced by the upstream CR pressure gradient. Finally the spectrum has two very interesting features at low energies. First, the computed numerical distribution is normalized to $f_{0}$ which is the amplitude of the test particle spectrum at $p_{i}$ that would have been produced by the subshock alone. In a similar vein the dotted line gives the slope $\left(-q_{s}\right)$ of the spectrum that would be produced if subshock compression were the only means of acceleration. Since $f\left(p_{i}\right) \simeq f_{0}$ and $(\partial \ln f / \partial \ln p) \simeq-q_{s}$ this implies that test particle theory applied to the lowest energy particles at the subshock gives an adequate picture of the modified spectrum 
near $p_{i}$. That this would in fact be the case was anticipated by Völk et al. (1991). The highest energy CRs suffer more compression with a consequent flatter spectrum.

\section{CONCLUSIONS}

There are three main conclusions that can be drawn from this work. First, it is possible to treat a strongly momentum dependent $\kappa$ in a self-consistent numerical code. Second, with the acceleration timescale increasing at least linearly with mo- mentum the system can, even in the strong shock and high injection limit, reach a quasi steady state where the bulk parameters stop evolving. Third, the CR spectrum in a strongly modified shock at the lowest energies is determined, both in amplitude and slope, by test particle theory applied to the subshock.

I am grateful to H. J. Völk and L. O'C. Drury for many discussions on this subject.

\section{REFERENCES}

Achterberg, A., Blandford, R., \& Periwal, V. 1984, A\&A, 132, 97

Axford, W. I., Leer, E., \& McKenzie, J. F. 1982, A\&A, 111, 317

Axford, W. I., Leer, E., \& Skadron, G. 1977, Proc. 15th Internat. Cosmic-

Ray Conf. (Plovdiv), 11, 132

Bell, A. R. 1978a, MNRAS, 182, 147

1978b, MNRAS, 182, 443

1987, MNRAS, 225,615

Berezhko, E. G., \& Krymsky, G. F. 1988, Soviet Phys.-Uspekhi, 31, 27

Blandford, R. D., \& Eichler, D. 1987, Phys. Rep., 154, 1

Blandford, R. D., \& Ostriker, J. P. 1978, ApJ, 221, L29

Dorfi, E. 1990, A\&A, 234, 419

Drury, L. O'C. 1983, Rep. Prog. Phys., 46, 973
Drury, L. O'C, \& Völk, H. J. 1981, ApJ, 248, 344

Duffy, P. 1992, A\&A, 262, 281

Ellison, D. C., \& Eichler, D. 1984, ApJ, 286, 691

Ellison, D. C., Möbius, E., \& Paschmann, G. 1990, ApJ, 352, 376

Falle, S. A. E. G., \& Giddings, J. R. 1987, MNRAS, 225, 399

Heavens, A. F. 1984, MNRAS, 210, 813

Jones, F. C., \& Ellison, D. C. 1991, Space Sci. Rev., 58, 259

Jones, T. W., \& Kang, H. 1990, ApJ, 363, 499

Kang, H., \& Jones, T. W. 1991, MNRAS, 249, 439

Krymsky, G. F. 1977, Dok. Akad. Nauk. SSR, 234, 1306

Skilling, J. 1975, MNRAS, 172, 557 